The eye was seriously damaged; X-ray showed the foreign body to be in the globe; in fact two foreign bodies. (1) The pellet. (2) A much smaller opaque substance which was not found at operation.

The eye was not removed until April 18 as there was some delay in obtaining permission from the parents. Immediately after removal, I opened the eye and found the vitreous infected. The pellet was found in the vitreous and close to it, and seemingly attached to it, were two cilia. The presence of the cilia in the vitreous is unique in my experience, but although it has been noted, it is sufficiently rare to make it worthy of being recorded again.

Yours faithfully,

Belfast,

W. A. ANDERSON.

July 26, 1939.

\title{
OCULAR DOMINANCE IN RELATION TO DEVELOPMENTAL APHASIA
}

To the Editors of THE BRITISH JOURnAL OF OPHTHALMOLOGY.

DEAR SIRS, - May I draw your attention to points arising out of the review you published of my monograph "Ocular Dominance in relation to Developmental Aphasia" in the current number of your Journal.

The account given there by your reviewer is an exceedingly inaccurate one as well as misleading, and one must conclude that he was able to give this quite sincere piece of work only very cursory perusal before writing his notice.

I shall take up the inaccuracies of statement one by one as they occur.

1. I am attached by your reviewer to the Physiology Department of Edinburgh University, whereas I am on the staff of the Psychology Department-this would give change of direction of emphasis to the investigation described.

2 . It is stated that "reading disability in 383 children" is investigated, whereas it is only incidence of disability within this total group that is investigated.

3. It is stated that the mean Intelligence Quotient of this group of 383 children is 100 with probable normal range of intelligence, whereas these findings refer to a smaller group of 49 children who show disability and handicap and are within the larger group.

4. The abbreviated form for Reading Accomplishment Quotient is misquoted as $\mathrm{R} \mathrm{A}$, a non-existent term in mental measurement. These are probably more trivial points, but 
5. A rather serious misstatement is made in paragraph 3 of the notice which begins "Only four disability children . . . ." Here two statements made, pp. 48,30 , in quite different reference are brought together, and further, the second of these statements has been misquoted. My own statement (p. 30) is that left-eyedness and reading disability run in families, not left-handedness as your reviewer has it. He therefore has created himself the absurdity to which he draws attention.

6. The final paragraph is, of course, irrelevant to the research and its findings, and arises out of a misunderstanding as to the sense in which the term "ocular dominance" is to be understood in this monograph. On p. 17 especial emphasis is given to this"It must be emphasised that all three tests (i.e., the tests of eyedness used in this research) are sighting or directional tests of eyedness. In view of the width of the term 'ocular dominance' it is essential to be clear in what sense we will use this term with reference to our findings."

Again on p. 28 the reader is reminded of the nature of the tests used.

Dr. Sampson's finding as to dominance (p. 59) refers solely to tests for retinal rivalry, and no correspondence of results is to be expected from tests by which quite different characters are explored. Put more definitely no correspondence is found between results from tests for retinal rivalry and from sighting or directional tests for eyedness. Dr. Sampson himself warns the reader to avoid such confusion:

Dr. Sampson examined 36 of the 49 disability cases, and his negative finding is of extreme importance being as it is in complete keeping with what, in consideration of our diagnosis of "developmental aphasia," we had hoped he would find was the case:

The issues involved are not noted by your reviewer, and hence his notice will fail to gain the attention of readers likely to be interested in the monograph. These might very briefly be mentioned.

The diagnostic value of left-eyedness for a very specialised pattern of language difficulty which would appear to be sex influenced:

A new outlook on the problem of severe backwardness in our schools, the aphasic pattern of difficulty not incidental causes or low mental capacity creating the whole "backward" situation.

The interesting genetic situation underlying developmental aphasia and which is now being more adequately explored; I refer here to the finding that heavy incidence of twinning exists in families in which the aphasia occurs.

This makes its contribution to investigation and understanding of ground causes for cerebral sidedness and laterality problems. The important facts remain as Ocular Dominance (understood as defined in this monograph) pointing to issues of cerebral sidedness, 
which in turn gives rise to developmental aphasic characters arising out of disturbance of mental functioning.

Before publication the monograph had the perusal and appreciation of men of high standing in the field entered by the research.

The review as published will certainly tend to repel rather than to attract interest for this research and its findings:

I can only see in it suggestions that here is a monograph somewhat slight, somewhat obvious, and in fact fairly considerably stupid.

I would therefore beg to lodge this truly sturdy protest.

$$
\text { Yours faithfully, }
$$

Psychology Department,

A. MaCMEEKEN.

UNIVERSITY OF EDINBURGH.

A ugust 8, 1939.

The Reviewer regrets the errors pointed out by Miss MacMeeken with regard to "Physiology" and "R. A." (which should of course be A. Q.), and also to the quotation from p. 30, which, as Miss MacMeeken rightly points out is meaningless as recorded, and should run "left-eyedness and reading disability run in families, and in such families there is heavy incidence of left-handedness." He had no wish to "repel interest for this research," and he hopes that both the monograph and Miss MacMeeken's comments will be read and studied.

\section{NOTES}

Corrigendum Addendum
Dr. CARL Hamburger, whose paper appeared in the August number of this Journal, requests that the part of the title referring to the author's town be changed to "formerly Berlin, temporarily Geneva." $\mathrm{He}$ also wished the following footnote to be inserted:- "This article was completed by the end of 1938, the author who lived in Germany until July, 1939, had no library facilities afforded him in which to give consideration to recent publications in other countries on this subject."

The 75th Anniversary WE have received from Dr. Harry Friedenwald
of the Foundation of the American Ophthal- a small brochure giving an account of the origin mological Society and first meeting of the American Ophthalmological Society. This is a reprint of an article by Dr. H. D. Noyes, of New York, the Recording Secretary. It was first printed in 1875 , and was reprinted for the commemorative meeting in June last. The Society is a very old foundation. It took its rise in the first place by means of correspondence between Hasket Derby, of Boston, and Drs. Noyes and Bumstead, of New York. 